\title{
A Comprehensive Protocol to Mitigate Inappropriate Medication in the Elderly
}

\author{
Vera Vlahović-Palčevski \\ Unit for Clinical Pharmacology, University Hospital Rijeka, Croatia.
}

Received: $10 / 08 / 2015$

Accepted: 19/09/2015

Published: 24/09/2015

How to cite this article: Vlahovic-Palcevski V. A comprehensive protocol to mitigate inappropriate medication in the elderly. EJCRIM 2015;2:DOI: 10.12890/2015_S1SP05

Conflicts of Interests: The authors declares that she has received speaker fees from Pfizer.

Acknowledgements: This abstract has been presented as a lecture at $2^{\text {nd }}$ International Seminar REPOSI: Targeting the burden of polypharmacy in the elderly.

This article is licensed under a Commons Attribution Non-Commercial 4.0 License

The percentage of elderly population is increasing and it is estimated that by $205030 \%$ of the population in developed countries will be over 65 . Elderly often have multiple chronic diseases that require multiple medications. Prescribing for older patients presents a unique challenge. Pre-marketing drug trials often exclude geriatric patients and approved drugs and doses may not be appropriate for older adults. Many medications need to be used with special caution because of age-related changes in pharmacokinetics (i.e., absorption, distribution, metabolism, and excretion) and pharmacodynamics.

Potentially inappropriate prescribing in older people is associated with increases in morbidity, hospitalization and mortality. Potentially inappropriate medications (PIMs) are defined as drugs with a potential risk that is higher than their potential benefit, particularly when safer alternative therapies exist.

Most screening tools for detecting PIMs list drugs that should be avoided in the elderly, some taking into account certain conditions. They assess quality of prescribing by analyzing large prescription databases and indicate where improvements should be made.

Clinically relevant approaches to avoid inappropriate prescribing in older adults include educational interventions, computerized order entry and decision support, multidisciplinary team care led by physicians, clinical pharmacists/pharmacologists, and combinations of these approaches.

A comprehensive protocol grouping PIMs into drugs with unfavorable benefit/risk ratio, drugs with questionable efficacy, drugs to be avoided with certain conditions and potentially serious drug-drug interactions may offer a clinical decision support to practicing physicians. 